\title{
Gaze related enhancement of hemispheric blood flow in a stroke patient
}

\author{
Stephen E Nadeau, Bruce Crosson, Ronald L Schwartz, Kenneth M Heilman
}

Geriatric Research, Education and Clinical Center

S E Nadeau

Department of

Veterans Affairs

Medical Center, the

Department of

Neurology, University

of Florida College of

Medicine, USA

$S$ E Nadeau

B Crosson

R L Schwartz

K M Heilman

Department of Clinical and Health

Psychology, University

of Florida College of

Health Professions,

Gainesville, Florida,

USA

$S$ E Nadeau

B Crosson

K M Heilman

Correspondence to:

Dr Stephen E Nadeau

GRECC-182, DVA Medical

Center, Gainesville,

FL 32608-1197, USA

Received 3 May 1996

and in revised form

15 October 1996

Accepted 23 December 1996

\begin{abstract}
Objective-A patient with a right posterior cerebral artery territory infarction and a left superior quadrantanopia exhibited improvement on various visual tasks including Goldmann perimetry during extreme right gaze. The phenomenon was investigated by functional imaging of cerebral blood flow.
\end{abstract}

Methods- ${ }^{99 \mathrm{~m}}$ Tc]HMPAO SPECT was carried out while the patient gazed to the right and to the left at an $8 \mathrm{~Hz}$ flash stimulus.

Results-When compared with left gaze, photic stimulation during right gaze was associated with an up to $39.8 \%$ increase in regional cerebral blood flow in the damaged right hemisphere, including Brodmann's areas 3-1-2, 7, 21, 22, 39, and 40.

Conclusions-These gaze related alterations in function and synaptic activity suggest the engagement of a novel arousal-like mechanism that may account in part for comparable findings in patients with neglect and other disorders, and may have relevance to rehabilitation.

(F Neurol Neurosurg Psychiatry 1997;62:538-540)

Keywords: neglect; cerebral blood flow; hemianopia

Neglect is the failure to report, respond, or orient to novel or meaningful stimuli presented to the side opposite a brain lesion, when this failure cannot be attributed to either sensory or motor deficits. ${ }^{1}$ Various data indicate that neglect is fundamentally a hemispatial phenomenon. One of the best ways to optimise patients' performance is to have them function in the preserved hemispace. Kooistra and Heilman, ${ }^{2}$ for example, noted that in a patient with hemispatial neglect and hemianopia due to an infarct of the right thalamus and adjacent occipitotemporal white matter, the hemianopia resolved when the patient directed his gaze to the right. They suggested that the hemianopia resolved because with right gaze, both retinotopic hemifields were moved into the patient's preserved hemispace. This hypothetical mechanism provides a plausible expla- nation for improvement of visual neglect wherein retinotopic fields can be dissociated from hemispatial fields but it does not provide an explanation for improvement of motor function of the upper limb by moving the limb into the preserved hemispace, ${ }^{3}$ or for improvement in contralateral touch and position detection by vestibular or optokinetic stimulation. ${ }^{4}$ Coslett $e t a l^{6}$ reported a patient with a left hemispheric stroke and mild neglect in whom several left hemispheric specific functions, including right upper limb movement, right hand tactile sensation, and language improved when performance was in the left (ipsilesional) hemispace or when the head was turned to the left. Coslett et al argued that this phenomenon could best be explained on the basis of stroke induced limitation in the efficiency or capacity for contemporaneous engagement of multiple left hemispheric subsystems. An alternative explanation is that ipsilesional gaze enhanced the function of the lesioned hemisphere. We report here results of cerebral blood flow (CBF) studies in a patient previously described by us $^{7}$ that provide support for this alternate, hemispheric enhancement hypothesis.

A 61 year old, high school educated man sustained a right occipitotemporal infarction (documented by MRI). Neurological examination was normal except for a dense homonymous left superior quadrantanopia and visual extinction in the left inferior quadrants on bilateral simultaneous stimulation. There was no evidence of hemispatial neglect. During the week after the stroke, further testing of visual fields was carried out using four methods: (1) detection of finger movement; (2) object naming; (3) shape identification; and (4) colour naming. For each modality, the patient gazed straight ahead, and about $30^{\circ}$ to the right or $30^{\circ}$ to the left, while maintaining his head in alignment with his body. The patient responded correctly to essentially all stimuli presented to the right visual field, regardless of direction of gaze. He responded correctly to only two of 60 stimuli presented to the left upper quadrant during left or centre gaze but to 36 of $\mathbf{4 5}$ of the stimuli presented during right gaze. He responded correctly to 17 of 60 stimuli presented to the left lower quadrant during left or centre gaze and his performance improved to 41 of 45 during right gaze. 
Profile of counts as a function of direction of gaze, $(A)$ right hemisphere, (B) left hemisphere, (C) right and left hemispheres during contrahemispheric gaze, and (D) right and left hemispheres during ipsihemispheric gaze.
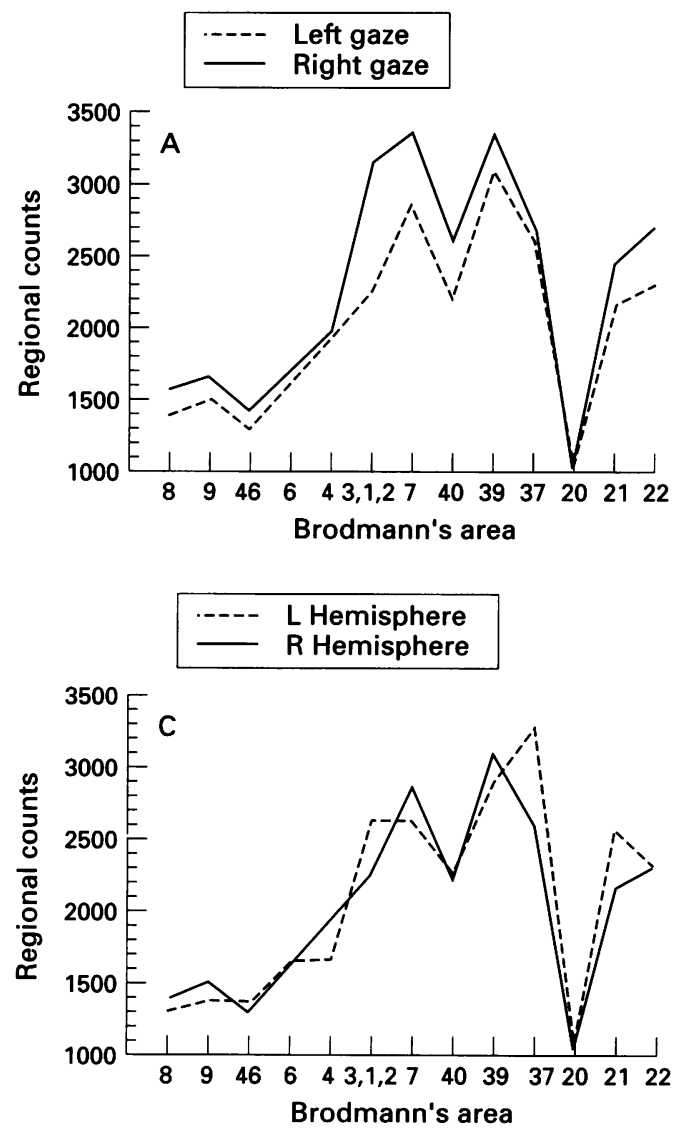

Methods

Informed consent was obtained under protocols approved by the Institutional Review Board of the University of Florida. Twenty months after the stroke, the patient underwent two $\left[{ }^{99 \mathrm{~m}} \mathrm{Tc}\right]$-hexamethylpropyleneamineoxime (HMPAO) SPECT studies of CBF, one during $45^{\circ}$ left (contralesional) gaze at an $8 \mathrm{~Hz}$ stroboscopic stimulus and the second three days later during $45^{\circ}$ right (ipsilesional) gaze at the same stimulus. The stimulus was provided by a PS33 photo stimulator (Grass Instruments, Quincy, MA, USA) with a $24 \mathrm{~cm}$ diameter lamp face, $125 \mathrm{~cm}$ distant from the subject's eyes; $10 \mu \mathrm{s}$ flash duration, peak intensity 380000 lumens. The subject was instructed to focus on a $3 \mathrm{~mm}$ diameter circular black mark at centre of the lamp face. The visual stimulus was presented for six minutes. T1 weighted MRI (Magnetom 1.5 Tesla scanner, Siemans, Iselin, NJ, USA) was also done to aid in SPECT image analysis. SPECT and MR images were processed as previously described.$^{8}$ Regions of interest were located on the surface of the MRI slices using a standard atlas $^{9}$ with some correction for individual variation in gyral anatomy. To sample a broad distribution of primary and association cortices, regions of interest values were obtained for the left and right hemispheres in Brodmann's areas $8,9,46,4,6,3-1-2,7,39,40,37,20$, 21 , and 22. Areas 17, 18, and 19 were not sampled because in the right hemisphere they were substantially involved in the infarct. Data were analysed using SYSTAT (Evanston, IL, USA).
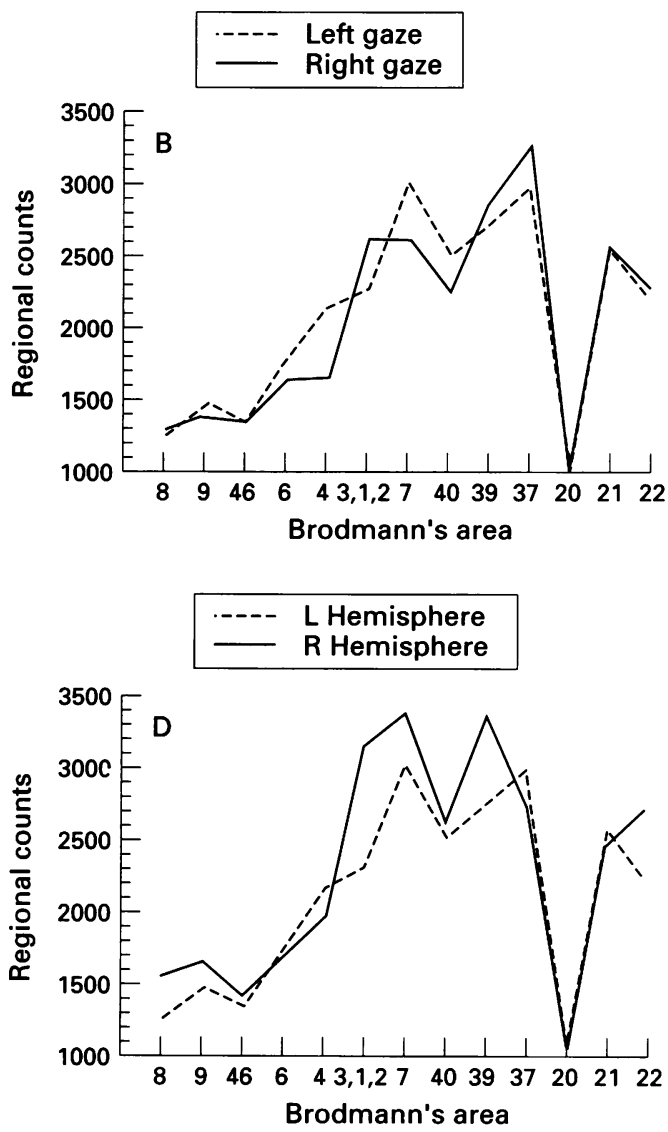

\section{Results}

Mean (SD) left hemispheric counts/voxel for the 13 regions of interest was 2114 (665) during left gaze and 2072 (715) during right gaze. Mean right hemispheric counts/voxel during left gaze was 2023 (625) and during right gaze 2290 (779). A $2 \times 2$ (hemisphere $\times$ gaze direction) repeated measures analysis of variance (with individual regions of interest counts/voxel serving as the dependent measure) showed no hemispheric effect $(F=0 \cdot 898, \mathrm{P}=$ $0.362)$, a strong gaze trend $(F=4 \cdot 185, \mathrm{P}=$ 0.063 ), and a highly significant interaction effect $(F=14.375, \mathrm{P}=0.003)$. This interaction effect was due entirely to gaze related alterations in right hemispheric CBF ( $t=3.906, \mathrm{P}=0.002)$; gaze related changes in left hemispheric CBF were not significant $(t=0.614, \mathrm{P}=0.551)$. The major changes in right hemispheric CBF were in areas 3-1-2, 7, $21,22,39$ and 40 in which there was a mean increase of $19 \cdot 2 \%$ (range $8 \cdot 4-39 \cdot 8 \%$ ) during ipsilateral (ipsilesional ) gaze (figure, A). There was no recognisable pattern of change in the left hemisphere (figure, B). The CBF in the two hemispheres was generally comparable during contralateral gaze (figure, $\mathrm{C}$ ), so the change in right hemispheric CBF during ipsilateral gaze represented an increase over normal (as defined by left hemispheric $C B F$ ), rather than a rise from below normal (figure, D).

\section{Discussion}

The major determinant of CBF is synaptic activity. ${ }^{10}$ Thus our studies show an increase in 
right hemispheric synaptic activity in areas 3-1-2, 7, 21, 22, 39 and 40 during ipsilesional gaze that was associated with improvement in visual function in the left visual field, particularly the superior quadrant. This change in CBF seems to reflect an increase over normal values (unless both hemispheres were hypoperfused during left gaze), rather than compensation of subnormal levels.

Whether the change was induced by gaze, the intention or plan to direct gaze, or some other related factor remains to be determined. The finding of Coslett $e t a l^{6}$ that improvement in function could be achieved by performance of a task in ipsilesional hemispace or head turning into ipsilesional space suggests that the ipsilesional direction of intention (which is required in all three manoeuvres) was the essential factor. The mechanism of the change is uncertain. An interhemispheric, transcallosal compensatory process seems most likely but other mechanisms involving the brainstem (midbrain reticular formation, superior colliculus), the thalamus, or the nucleus basalis are possible. ${ }^{11}$ The broad distribution of the changes suggests a process more akin to arousal-that is, a relatively non-specific increase in activity over cortices subserving various functions, rather than an attentional process, in which case we might have expected changes limited to cortices implicated in visual processing, such as areas $7,20,21,37$, and 39.

These findings suggest not only an unreported brain mechanism, but the possibility of a compensatory mechanism that might be important in rehabilitation. The alterations in CBF described in this patient were induced by extreme ipsilesional gaze, an intrinsically dysfunctional manoeuvre in a patient with hemispatial neglect because it exaggerates the neglect of contralesional hemispace at the same time that it may improve function in ipsilesional hemispace. However, it may be possible to engage this compensatory mechanism by other means. For example, hemispatial neglect may be reduced by cold caloric stimulation of the contralateral vestibular apparatus, ${ }^{12-14}$ optokinetic stimulation moving in the contralateral direction, ${ }^{4}{ }^{15}$ contralateral vibratory stimulation of the neck, ${ }^{12}$ and use of the contralesional limb in contralesional hemispace. ${ }^{16-19}$ Various explanations have been offered to account for these findings but the activation hypothesis, which our data support, could account in whole or part for all of them.

This research was supported by a grant from the Merit Review Program of the Department of Veterans Affairs. We are gratefu to Morris Thompson for his technical assistance.

1 Heilman KM, Watson RT, Valenstein E. Neglect and related disorders. In: Heilman KM, Valenstein E, eds. Clinical neuropsychology. 3rd ed. New York: Oxford,

2 Kooistra CA, Heilman KM. Hemispatial visual inattention masquerading as hemianopia. Neurology 1989;39: $1125-7$.

3 Meador K, Watson RT, Bowers D, Heilman KM. Hypometria with hemispatial and limb motor neglect. Brain 1986;109:293-305

4 Vallar F, Antonucci G, Guariglia C, Pizzamiglio L. Deficits of position sense, unilateral neglect and optokinetic stim ulation. Neuropsychologia 1993;31:1 191-200.

5 Vallar G, Bottini G, Rusconi ML, Sterzi R. Exploring somatosensory hemineglect by vestibular stimulation. Brain 1993;116:71-86.

6 Coslett HB, Schwartz MF, Goldberg G, Haas D, Perkins J. Multi-modal hemispatial deficits after left hemisphere Multi-modal hemispatial deficits
stroke. Brain 1993;116:527-54.

7 Nadeau SE, Heilman KM. Gaze-dependent hemianopia without hemispatial neglect. Neurology 1991;41:1244-50.

8 Crosson B, Williamson DJG, Shukla SS, Honeyman JC, Nadeau SE. A technique for localizing activation in the human brain with [ $\left.{ }^{99 \mathrm{~m}} \mathrm{Tc}\right]-\mathrm{HMPAO}$ SPECT: a validation study using visual stimulation. $f \mathrm{Nucl}$ Med 1994 35:755-63.

Damasio H, Damasio AR. Lesion analysis in neuropsychology. New York: Oxford University Press, 1989.

10 Nadeau SE, Crosson B. A guide to the functional imaging of cognitive processes. Neuropsychiatry, Neuropsychology, and Behavioral Neurology 1995;8:143-62.

11 Sato $\mathrm{H}$, Hata $\mathrm{Y}$, Masui $\mathrm{H}$, Tsumoto T. A functional role of cholinergic innervation to neurons in the cat visual cortex. $\mathcal{F}$ Neurophysiol $1987 ; 58: 765-80$.

12 Karnath H-O. Subjective body orientation in neglect and the interactive contribution of neck muscle propriocepthe interactive contribution of neck muscle propriocep-

13 Vallar G, Sterzi R, Bottini G, Cappa S, Rusconi L. Temporary remission of left hemianesthesia after vestibuar stimulation: a sensory neglect phenomenon. Cortex 1990;26:123-31.

14 Rubens AB. Caloric stimulation and unilateral visual neglect. Neurology 1985;35:1019-24.

15 Pizzamiglio L, Frasca R, Guariglia C, Incoccia C Antonucci $\mathrm{G}$. Effect of optokinetic stimulation in patients with visual neglect. Cortex 1990;26:535-40.

16 Joanette Y, Brouchon M, Gauthier L, Samson M. Pointing with left $v$ right hand in left visual field neglect. Neuropsychologia 1986;24:391-6.

17 Halligan PW, Marshall JC. Laterality of motor response in visuospatial neglect: a case study. Neuropsychologia 1989 , 27:1301-7.

18 Robertson IH, North N. Spatio-motor cuing in unilateral left neglect: the role of hemispace, hand and motor acti-

19 Robertson IH, North N. Active and passive activation of left limbs: influence on visual and sensory neglect. Neuropsychologia 1993;31:293-300. 